\title{
Validación de SYBR-14 y 6-CFDA para Evaluar la Viabilidad e Integridad de la Membrana Plasmática en Espermatozoides Caninos de Raza Chihuahua
}

\author{
Validation of SYBR-14 and 6-CFDA to Evaluate the Viability and \\ Plasma Membrane Integrity in Sperm of Chihuahua Canine Breed
}

\author{
Paulo Salinas $^{*, * *}$; Felipe Pezo*; Raúl Sánchez ${ }^{* * * *}$ \& Jennie Risopatrón ${ }^{* * * *}$
}

SALINAS, P.; PEZO, F.; SÁNCHEZ, R. \& RISOPATRÓN, J. Validación de SYBR-14 y 6-CFDA para evaluar la viabilidad e integridad de la membrana plasmática en espermatozoides caninos de raza Chihuahua. Int. J. Morphol., 32(1):16-21, 2014.

RESUMEN: En la aplicación de técnicas reproductivas es importante determinar in vitro la capacidad fecundante de los espermatozoides, para ello se utilizan combinaciones de tinciones para evaluar los diferentes parámetros de función espermática, aumentando así la precisión de la estimación de la muestra. El objetivo del estudio fue comparar la efectividad de la utilización de los fluorocromos 6-CFDA y SYBR-14 combinados con PI para determinar la viabilidad e integridad de la membrana plasmática por citometría de flujo. Se utilizó semen fresco de caninos $(\mathrm{n}=5)$ de raza Chihuahua, con una concentración espermática $>150 \times 10^{6}$ esp/ml y motilidad progresiva $>80 \%$. Tres protocolos fueron ensayados: grupo 1: SYBR-14/PI, grupo 2: 6-CFDA/PI y grupo 3: PI. La integridad de la membrana plasmática de los espermatozoides fue similar entre grupos 1 y 2, independiente del fluorocromo utilizado $(37,26 \pm 13,9$ y 33,8 $\pm 14,6$, respectivamente; $p=0,4601)$. Asimismo, la viabilidad espermática entre los grupos 1,2 y $3(62,7 \pm 13,9,66,1 \pm 14,6$ y $66,4 \pm 13,3$, respectivamente; $\mathrm{p}=0,8987$ ). En conclusión, no se evidenció diferencias en la efectividad para determinar la viabilidad e integridad de la membrana plasmática mediante la utilización de SYBR-14 y 6-CFDA, ambas tinciones pueden ser incorporadas al análisis de rutina de semen canino de raza Chihuahua.

PALABRAS CLAVE: Caninos; Chihuahua; Semen; Espermatozoides; SYBR-14; 6-CFDA; Citometría de flujo.

\section{INTRODUCCIÓN}

Tradicionalmente, los análisis de laboratorio utilizados para evaluar la calidad de una muestra de semen previo a la inseminación artificial en caninos implican la evaluación de varios parámetros a nivel funcional, esto es debido a que los espermatozoides son células complejas que requieren cumplir con una serie de modificaciones con el objetivo de lograr la fecundación, especialmente a nivel de membrana plasmática. Por lo tanto, los estudios que mejoren y estandaricen la evaluación de éste parámetro son de interés, más aún cuando se asocian a una especie en particular.

La integridad de la membrana plasmática es uno de los parámetros evaluados con mayor frecuencia durante el análisis seminal de rutina y su determinación es útil para predecir in vitro la capacidad fecundante del es- permatozoide (Rijsselaere et al., 2005). La membrana plasmática del espermatozoide es esencial para algunas funciones metabólicas tales como la capacitación, interacción con células de la tuba uterina, reacción del acrosoma e interacción con la zona pelúcida, por lo tanto, la pérdida de su integridad es considerada como incompatible con la capacidad del espermatozoide de fecundar un ovocito in vivo.

La técnica ideal para evaluar cualquier parámetro espermático debe ser rápida, específica y automatizada. Las técnicas para evaluar la membrana plasmática se basan en el incremento de la permeabilidad a sustancias, tales como sondas fluorescentes, con capacidades diferentes para atravesar una membrana íntegra. Proteínas espe-

\footnotetext{
Laboratorio de Anatomía Veterinaria, Escuela de Medicina Veterinaria, Facultad de Recursos Naturales y Medicina Veterinaria, Universidad Santo Tomás, Temuco, Chile.

** Programa de Doctorado en Ciencias Morfológicas, Fac. Medicina, Universidad de La Frontera, Temuco, Chile.

*** Centro de Biotecnología en Reproducción (CEBIOR-BIOREN), Fac. Medicina, Universidad de La Frontera, Temuco, Chile. Financiamiento: DI12-0089 Dirección de Investigación, Universidad de La Frontera.
} 
cíficas de la membrana plasmática facilitan el transporte de glucosa y fructosa desde el ambiente extracelular al interior del espermatozoide (Schürmann et al., 2002). Las pruebas basadas en fluorescencia utilizan fluorocromos impermeables solos o combinados con tinciones fluorescentes permeables (6-CFDA, SYBR-14) (Garner et al., 1986; Harrison \& Vickers, 1990; Garner \& Johnson, 1995; Pintado et al., 2000; Martínez-Pastor et al., 2004). Los espermatozoides con membranas plasmáticas íntegras excluyen a aquellas tinciones impermeables, las cuales penetran y tiñen específicamente las células muertas. Esto es evidenciado en la tinción selectiva y emisión dual de colores que permite la cuantificación objetiva de células viables y no-viables en un citómetro de flujo. Diferentes tinciones son utilizadas en el análisis seminal de caninos para evaluar las funciones celulares del espermatozoide, tanto estructural como bioquímicas. Tinciones específicas de fluorocromos proveen información cuantitativa, relativa a la integridad y permeabilidad de la membrana plasmática, que discrimina entre un espermatozoide funcional de otro no-funcional, aumentando así la precisión de la estimación de la muestra.

El ioduro de propidio (PI), es una tinción impermeable fluorescente ADN-específica, que combinada con fluorocromos permeables, tales como SYBR-14 (Silva \& Gadella, 2006) o 6-CFDA (6-carboxiefluoresceína diacetato) (Kim et al., 2010a, 2010b), actúan como marcadores de la integridad de la membrana plasmática (Brito et al., 2003). La combinación de SYRB-14 con PI es una de las tinciones más utilizadas para determinar la viabilidad e integridad de la membrana plasmática (Garner \& Johnson). Ambas se unen al ADN, pero difieren en su capacidad de penetrar la membrana plasmática (Peña et al., 2006).

SYBR-14 es un colorante de membrana acilado, no fluorescente y permeable a la membrana plasmática, el cual puede entrar en los espermatozoides, siendo desacilado por las esterasas intracelulares, convirtiéndose en un fluoróforo verde que es retenido intracelularmente por las membranas plasmáticas intactas (Silva \& Gadella; Peña et al., 1999). Una vez que ocurre la muerte celular, ésta pierde la capacidad para resistir el influjo de tinción fluorescente roja del PI, reemplazando de esta manera al SYBR-14 (Garner \& Johnson). Los espermatozoides viables con membrana plasmática intacta exhibirán fluorescencia verde (SYBR-14 + ), mientras que los espermatozoides con membrana plasmática dañada (muertos) se teñirán rojos por el PI (Peña et al., 1999; Rijsselaere et al., 2002). De esta manera, siempre los espermatozoides se unirán a una de las dos sondas de unión al ADN (PI o SYBR-14) permitiendo predecir su condición de viabilidad por medio de su emisión fluorescente (Nagy et al., 2003).
La combinación de 6-CFDA y PI provee información cuantificable del estado funcional del espermatozoide por sus características moleculares (Brito et al.). El 6-CFDA es un compuesto no fluorescente permeable a la membrana plasmática del espermatozoide, que es convertido por esterasas citoplasmáticas en carboxifluoresceína, un fluoróforo verde altamente fluorescente, impermeable a la membrana que queda atrapada intracelularmente en membranas intactas (Garner et al.). Debido a que se pierde rápidamente cuando la membrana está dañada, el 6-CFDA es una sonda sensible para evaluar la integridad de la membrana plasmática. El PI tiñe el ADN de células muertas o con daño en las membranas, y al excitarse produce una fluorescencia de color roja (Silva \& Gadella). La utilización de 6CFDA y PI permite hacer una distinción entre los espermatozoides, ya que se pueden identificar tres poblaciones celulares: vivos con membrana plasmática intacta, moribundos y muertos.

Antecedentes de diferencias entre fluorocromos en la efectividad de detección del estado funcional de la membrana plasmática no han sido reportados en caninos de raza Chihuahua. El objetivo del estudio fue validar, en espermatozoides caninos de raza Chihuahua, la utilización de fluorocromos permeables tales como SYBR-14 y 6-CFDA combinados con PI respecto a la efectividad de detección del estado funcional de la membrana plasmática analizados por citometría de flujo.

\section{MATERIAL Y MÉTODO}

Los ensayos se realizaron en los laboratorios del Centro de Biotecnología en Reproducción (CEBIOR), Facultad de Medicina, Universidad de La Frontera, Temuco, Chile.

Animales. De acuerdo a las normas establecidas por la Guide to the Care and Use of Experimental Animals (Olfert et al., 1998) se utilizaron machos caninos $(\mathrm{n}=5)$ adultos (Canis lupus familiaris), raza Chihuahua, pelo largo y corto, con peso promedio de $2,5 \mathrm{~kg}$, y cuyas edades fluctuaron entre 1 y 4 años. Los animales pertenecen al criadero de perros Chihuahua Monte Blanco, Temuco, Chile. La condición sanitaria de los donantes fue evaluada periódicamente por un médico veterinario, y mantenidos con una dieta balanceada y agua ad libitum. Para colectar los eyaculados los animales fueron entrenados previamente como donantes de semen y durante la etapa experimental se mantuvo un periodo mínimo de tres días entre cada colecta (Kutzler, 2005).

Obtención y evaluación inicial del eyaculado. Espermatozoides de 5 eyaculados diferentes obtenidos de 5 
machos fueron colectados y procesados según lo descrito por Kutzler. Se colectó la segunda fracción rica en espermatozoides en diferentes vasos estériles previamente temperados a $38^{\circ} \mathrm{C}$, y fueron transportados al laboratorio en un contenedor termo aislado. Se realizó un análisis de cada eyaculado que incluyó la evaluación de la concentración y motilidad. La concentración fue determinada utilizando un fotómetro SDM1 (SpermaCue, Minitüb, Tiefenbach, Germany). La motilidad progresiva fue determinada por dos observadores para disminuir la subjetividad, utilizando un microscopio de luz (Axiolab drd, KT 450905, Zeiss) con platina temperada $\left(38^{\circ} \mathrm{C}\right)$ y condensador de contraste de fases a 400X. El porcentaje de espermatozoides mótiles se determinó observando un mínimo de 300 células, por lo menos en seis campos diferentes, un promedio de seis estimaciones sucesivas fue registrado como la motilidad final. El porcentaje de espermatozoides con motilidad progresiva se determinó considerando los grados 3 y 4 de una escala de 0 a 4 (0: sin movimiento, 1: movimiento sin avance, 2 : movimiento en círculo, 3: movimiento progresivo rectilíneo suave, 4: movimiento rectilíneo rápido (Risopatrón et al., 2002; Kim et al., 2010a). El criterio de inclusión de los eyaculados al estudio fue aquellos que cumplieron con las siguientes características: volumen de segunda fracción del eyaculado mayor a 1,5 ml, concentración mayor a 150x $10^{6}$ espermatozoides/ml y motilidad progresiva $>85 \%$.

Diseño experimental. Para evaluar la efectividad de los fluorocromos 6-CFDA y SYBR-14 combinados con PI en la determinación del estado funcional de la membrana plasmática analizados por citometría de flujo se realizaron los siguientes grupos experimentales: grupo1: SYBR-14/PI, grupo2: 6-CFDA/PI y grupo3: PI.

Viabilidad e integridad de membrana plasmática. Se evaluó utilizando dos tinciones: (a) kit comercial LIVE/DEAD® Sperm Viability Kit-Invitrogen (Salinas et al., 2013). Se preparó una solución stock diluyendo $1 \mu \mathrm{l}$ de SYBR-14 en 99 $\mu l$ de DMSO (dilución 1:100), se alicuotó y almacenó a $20^{\circ} \mathrm{C}$, se descongeló previo a su utilización. Un volumen conteniendo una cantidad de $2,5 \times 10^{6}$ espermatozoides $/ \mathrm{ml}$, fue mezclado con $250 \mu \mathrm{l}$ de Fosfato Buffer Salino (PBS, P4417Sigma, St. Louis MO, USA) se adicionó 0,5 $\mu 1 \mathrm{de}$ SYBR-14 (10 mM). Se incubó durante 7 minutos a $38^{\circ} \mathrm{C}, \mathrm{y}$ se adicionó $1 \mu \mathrm{l}$ de PI $(250 \mathrm{mg} / \mathrm{ml})$ e incubando nuevamente por otros 7 minutos a $38^{\circ} \mathrm{C}$, al final de este tiempo se adicionó $1 \mathrm{ml}$ de PBS para lavar y centrifugar a 300xg durante 5 minutos, se descartó el sobrenadante y las células se resuspendieron en $400 \mu \mathrm{l}$ de PBS para ser analizadas en el citómetro de flujo. Se analizaron 10.000 células aproximadamente. Los espermatozoides se clasificaron como: vivos con membrana plasmática intacta (SYBR-14+/PI-), muertos (SYBR-14-/PI+) y moribundos (SYBR-14+/PI+) (Fig.
1). (b) 6-CFDA/PI (Molecular Probes®) procesado según lo descrito por Kim et al. (2010b). Un volumen de suspensión espermática $\left(2,5 \times 10^{6}\right.$ espermatozoides $\left./ \mathrm{ml}\right)$ fue mezclado con $250 \mu \mathrm{l}$ de PBS, posteriormente se adicionó $1 \mu \mathrm{l}$ de 6 CFDA y $2 \mu \mathrm{l}$ de PI. Se incubó durante 15 minutos a $38^{\circ} \mathrm{C}$. Una vez finalizado el periodo de incubación se agregó $1 \mathrm{ml}$ de PBS para lavar por centrifugación a 300xg durante 5 minutos, se descartó el sobrenadante y las células fueron resuspendidas en $400 \mu \mathrm{l}$ de PBS para ser analizadas en el citómetro de flujo. Se evaluaron 10.000 células aproximadamente. Los espermatozoides analizados se clasificaron según lo descrito por Kim et al., (2010a): vivos con membrana plasmática intacta (CFDA+/PI-), moribundos (CFDA+/PI+) y muertos (CFDA-/PI+) (Fig. 1).

Citometría de flujo y obtención de datos. Las lecturas de la fluorescencia fueron realizadas en un citómetro de flujo FACS Canto II de Becton Dickinson. Para el uso del citómetro, los espermatozoides, fueron teñidos con cada una de las tinciones específicas. Cada análisis consistió en la adquisición de un mínimo de 10.000 espermatozoides teñidos, los cuales fueron cuantificados simultáneamente para cada fluorescencia. Se evaluaron 600-1000 células/s a través del láser. Fueron registrados los datos de 10.000 células las cuales fueron excitadas a $488 \mathrm{~nm}$ usando un láser de Argón. La fluorescencia fue detectada con un filtro de ancho de banda de $520 \mathrm{~nm}$ (FL1) y de $610 \mathrm{~nm}$ (FL3). Ambos sobre escalas logarítmicas. La digitalización de los datos se realizó utilizando el software BD Cell Quest ${ }^{\mathrm{TM}}$ Pro.

Análisis estadístico. El estudio fue de tipo experimental, descriptivo e inferencial. Los resultados fueron expresados como promedio \pm DE. Se utilizó la prueba de D’Agostino Pearson para detectar normalidad en los datos. Para detectar diferencias significativas en la determinación de viabilidad celular entre los grupos 1, 2 y 3 se utilizó la prueba de Varianza Paramétrico ANOVA (o su contraparte no paramétrica de Kruskall-Wallis). Posteriormente, para detectar diferencias significativas entre grupos se aplicó el Postest Paramétrico de Comparación Múltiple de Tuckey (o su contraparte no paramétrica de Dunnett). Para detectar diferencias significativas en la integridad de la membrana plasmática entre los grupos 1 y 2 se utilizó la prueba Paramétrica de Student (o su contraparte no paramétrica de U-Mann Whitney). Además, para proporcionar una medida de asociación lineal y para cuantificar la concordancia entre las mediciones individuales de cada técnica se determinó el coeficiente de correlación de Pearson e Interclase (ICC), respectivamente. Además, se calculó la variabilidad en la determinación de integridad de membranas en un mismo grupo con el Coeficiente de Variación (CV) expresado como porcentaje. La significancia estadística para todas las pruebas fue establecida para un $\mathrm{P}<0,05$. El análisis de los datos 
se realizó con el programa SPSSv.21 (IBM Corp. Released 2012. IBM SPSS Statistics for OSX, Version 21.0. Armonk, NY).
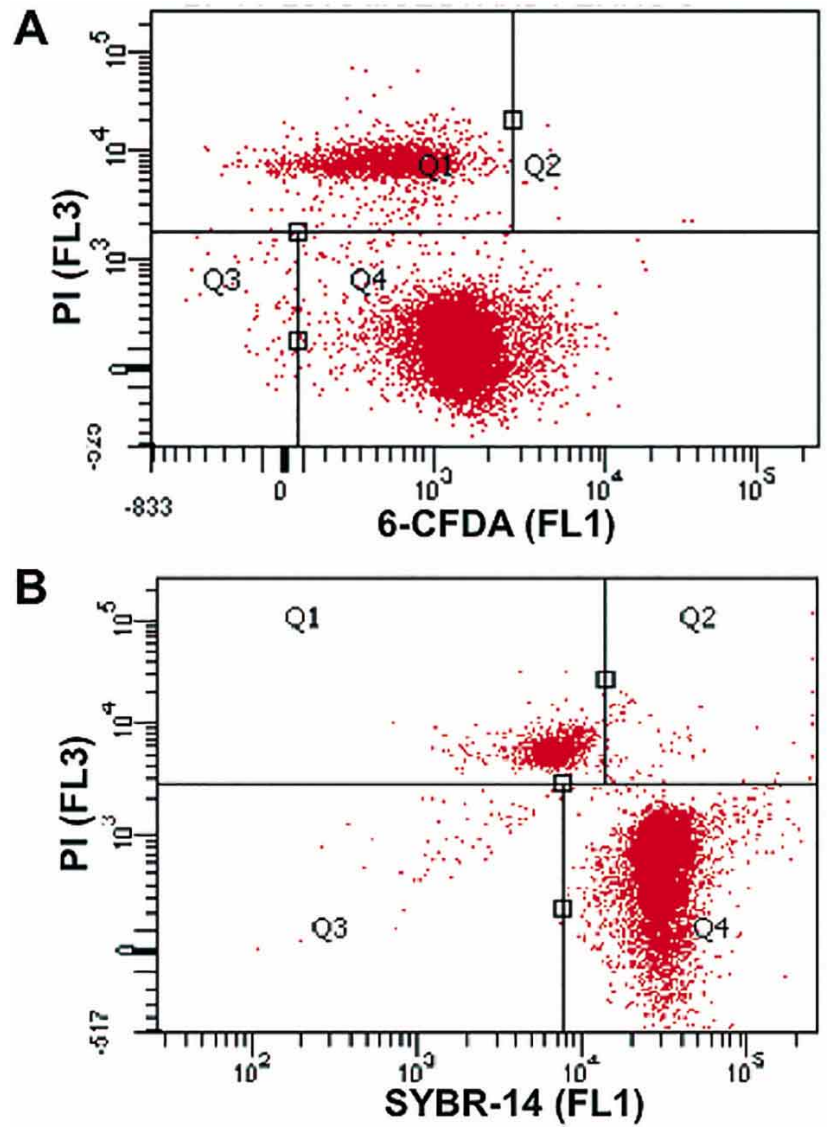

Fig. 1. Análisis por citometría de flujo de semen canino de raza Chihuahua. (A) Tinción 6-CFDA/PI. Q1: muertos (CFDA-/PI+), Q3: moribundos (CFDA+/PI+), Q4: vivos con membrana plasmática intacta (CFDA+/PI-). (B) Tinción SYBR-14/PI. Q1: no viables y membrana plasmática dañada (SYBR14-/PI+), Q2: espermatozoides moribundos (SYBR14+/PI+), Q4: espermatozoides viables con membrana plasmática intacta (SYBR14+/PI-).

\section{RESULTADOS}

El porcentaje promedio de espermatozoides caninos de raza Chihuahua viables con membrana plasmática ínte-

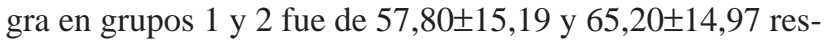
pectivamente (Fig. 2A). El análisis estadístico no detectó diferencias significativas entre las medias $(\mathrm{p}=0,4601)$. Un $2,9 \pm 1,5 \%$ y $0,6 \pm 0,3 \%$ de espermatozoides viables en grupos 1 y 2 , respectivamente, presentaron membrana plasmática dañada y fueron clasificados como moribundos. Respecto a la viabilidad espermática (Q3 + Q4; Fig. 1) el porcentaje promedio de espermatozoides caninos de raza Chihuahua viables en grupo 1 (SYBR14+/PI-), 2 (CFDA+/

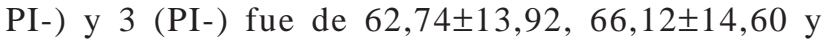
$66,44 \pm 13,34$, respectivamente (Fig. 2B). El análisis estadístico no detectó diferencias significativas entre las medias ( $\mathrm{p}=0,8987)$. Finalmente, la efectividad en la detección de membranas íntegras obtenidos con SYBR-14 se correlacionaron positivamente con los obtenidos con 6CFDA ( $r=0,9742 ; p=0,0049)$ y demostraron, además, una excelente concordancia $(\mathrm{r}=0,987)$ (Tabla I).

Tabla I. Análisis de correlación intraclase. a. El estimador es el mismo, ya esté presente o no el efecto de interacción. b. Coeficientes de correlación intraclase de tipo $\mathrm{C}$ utilizando una definición de coherencia, la varianza inter-medidas se excluye de la varianza del denominador. c. Esta estimación se calcula asumiendo que no está presente el efecto de interacción, ya que de otra manera no es estimable.

\begin{tabular}{|c|c|c|c|}
\hline & \multirow[b]{2}{*}{$\begin{array}{c}\text { Correlación } \\
\text { intraclaseb }\end{array}$} & \multicolumn{2}{|c|}{ Intervalo de confianza } \\
\hline & & $\begin{array}{l}\text { Límite } \\
\text { inferior }\end{array}$ & $\begin{array}{c}\text { Límite } \\
\text { superior }\end{array}$ \\
\hline $\begin{array}{l}\text { Medidas } \\
\text { individuales }\end{array}$ & $0,974 a$ & 0,776 & 0,997 \\
\hline $\begin{array}{l}\text { Medidas } \\
\text { promedio }\end{array}$ & $0,987 \mathrm{c}$ & 0,874 & 0,999 \\
\hline
\end{tabular}

B

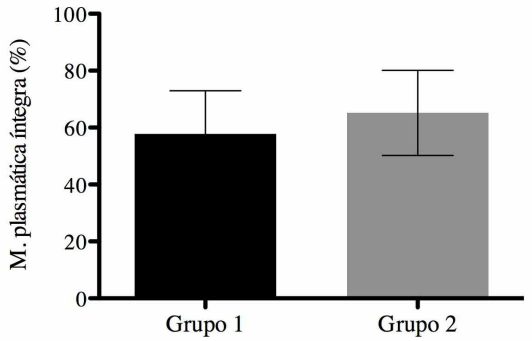

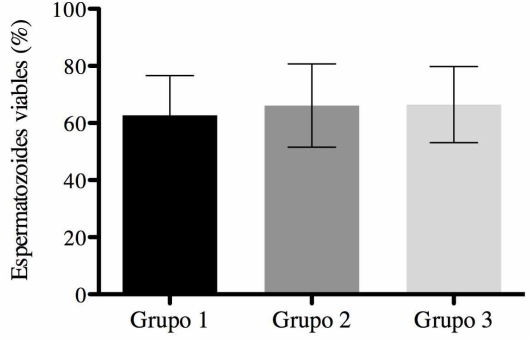

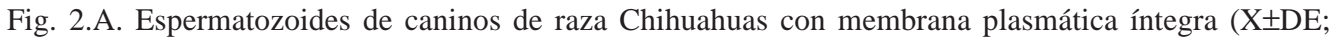
$\mathrm{p}=0,4601)$ y $\mathrm{B}$. viables (PI-) $(\mathrm{X} \pm \mathrm{DE} ; \mathrm{p}=0,8987)$. 


\section{DISCUSIÓN}

La combinación de PI con SYBR-14 o 6-CFDA ha sido utilizada efectivamente para evaluar poblaciones celulares vivas/muertas con membrana plasmática integra/dañada en semen de bovino, equino, canino, conejos y humano (Garner \& Johnson). Sin embargo, no ha sido descrita la comparación en la efectividad de detección de daño en la membrana plasmática del espermatozoide en caninos de raza Chihuahua. En el presente estudio, suspensiones de espermatozoides de caninos de raza Chihuahua se sometieron a tinciones con PI, solo y combinado con SYBR-14 o 6CDFA. Los resultados demostraron que las dos combinaciones de fluorocromos utilizadas para evaluar la integridad de la membrana del espermatozoide en caninos son efectivas, producen resultados estables y pueden ser utilizadas en herramientas automatizadas, como el citómetro de flujo y analizadas a través de un software.

Las dos principales ventajas de la utilización de SYBR-14 sobre las tinciones en base a enzimas, como el 6CFDA son: a) El tiempo de tinción no es crítico y la tinción de fondo (contraste) es prácticamente inexistente cuando se observan en un microscopio de fluorescencia (Garner \& Johnson) y b) Las tinciones en base a esterasas para evaluar integridad de la membrana, como es el caso del 6-CFDA, requieren mayor atención en el tiempo de procesado de las muestras debido a que la fluorescencia aumenta con el tiempo durante la observación en el microscopio de fluorescencia. $\mathrm{Al}$ contrario, el SYBR-14 rápidamente alcanza un equilibrio (menos de $15 \mathrm{~min}$ ) por lo tanto es más estable. El background en muestras teñidas con SYBR-14 es casi inexistente, por lo tanto, los artefactos son mínimos combinado con PI (Garner et al.).

Nuestros resultados en semen canino de raza Chihuahua fueron inferiores a los obtenidos en pool de se- men fresco de perros Pastor Alemán, Golden Retriever y Labrador con un $82,66 \pm 2,8 \%$ de espermatozoides con membrana plasmática íntegra y $8,4 \pm 2,6 \%$ de espermatozoides viables con membrana dañada evaluado por citometría de flujo (Cheuquemán et al., 2012). Además, fueron inferiores a los obtenidos mediante microscopía de fluorescencia y en diferentes razas de caninos, con membrana plasmática íntegra en un rango de 77-99\% (Volpe et al., 2009). Con respecto a la determinación de membrana plasmática íntegra con la tinción 6-CFDA nuestros resultados fueron superiores a los reportados en caninos de raza Beagle y Schnauzer (Kim et al., 2010b).

La medición por citometría de flujo de la integridad de las membranas plasmáticas con PI solo, o combinado con SYBR-14 o 6-CFDA no demostró diferencias en la detección de daño entre los grupos, con una correlación positiva entre ambas combinaciones de fluorocromos, similar a los obtenidos en estudios previos en otras especies (Brito et al.; Anzar et al., 2009) con una excelente concordancia entre cada una de las evaluaciones realizadas con ambos fluorocromos. En contraposición a estos resultados, la evaluación en semen de ovino con microscopia de fluorescencia de ambos fluorocromos combinados con PI mostraron diferencias en la detección de daño en membranas entre las combinaciones SYBR-14/PI y 6-CFDA/PI, argumentándose que la proporción de plasmalema intacto parece estar sobrestimado por SYBR-14/PI comparado con 6-CFDA/PI (Yaniz et al., 2013). La explicación a esta diferencia la atribuyen al tiempo de incubación a $37^{\circ} \mathrm{C}$ el cual no es necesario para el SYBR-14, en cambio para 6-CFDA se requieren 4 min.

En conclusión, no se evidenció diferencias en la efectividad para determinar la viabilidad e integridad de la membrana espermática mediante la utilización de SYBR-14 y 6CFDA, ambas tinciones pueden ser incorporadas al análisis de rutina de semen canino de raza Chihuahua.

SALINAS, P.; PEZO, F.; SÁNCHEZ, R. \& RISOPATRÓN, J. Validation of SYBR-14 and 6-CFDA to evaluate the viability and plasma membrane integrity in sperm of Chihuahua canine breed. Int. J. Morphol., 32(1):16-21, 2014.

SUMMARY: In applying reproductive techniques in vitro it is important to determine the fertilizing capacity of the sperm, for this a combination of dyes were used to assess different parameters of sperm function, thereby increasing the accuracy of the estimation of the sample. In dogs (Canis lupus familiaris) Chihuahua breed there is no precedent for evaluating sperm function parameters. The aim was to assess the viability and plasmatic membrane integrity, basic parameters of sperm function. Propidium iodide (PI) was used, a fluorescent dye-specific DNA, which combined with fluorochromes permeable acts as marker of the sperm membrane integrity. The effectiveness of the use of 6-CFDA and SYBR-14 fluorochromes combined with PI was also compared to determine viability and sperm membrane integrity using flow cytometry. Fresh semen of dogs $(\mathrm{n}=5) \mathrm{Chihuahua}$ breed was used with a concentration of $>200 \times 10^{6} \mathrm{sp} / \mathrm{ml}$ and progressive motility $>80 \%$. Three protocols were performed: group 1: SYBR-14/PI, group 2: 6-CFDA/PI and group 3: PI. The plasma membrane integrity of sperm was similar, independent of the fluorophore used between groups 1 and 2 (13.9 \pm 37.26 and $33.8 \pm 14.6$, respectively, $\mathrm{p}=0.4601)$. This also applied to sperm viability between groups 1,2 and $3(62.7 \pm 13.9,66.1 \pm 14.6$ and $66.4 \pm 13.3$, respectively, $\mathrm{p}=0.8987$ ). No difference was demonstrated in effectiveness to determine the viability and integrity of the sperm membrane using SYBR14 and 6-CFDA, both dyes can be incorporated in to routine analysis of semen in canine Chihuahua breed.

KEY WORDS: Canine; Chihuahua; Semen; Sperm; SYBR-14; 6-CFDA; Flow cytometry. 


\section{REFERENCIAS BIBLIOGRÁFICAS}

Anzar, M; Kroetsch, T. \& Buhr, M. M. Comparison of different methods for assessment of sperm concentration and membrane integrity with bull semen. J. Androl., 30:661-8, 2009.

Brito, L. F.; Barth A. D.; Bilodeau-Goeseels, S.; Panich P. L. \& Kastelic, J. P. Comparison of methods to evaluate the plasmalemma of bovine sperm and their relationship with in vitro fertilization rate. Theriogenology, 60(8):1539-51, 2003.

Cheuquemán, C.; Bravo, P.; Treulén, F.; Giojalas, L. C.; Villegas, J.; \& Sánchez, R. \& Risopatrón, J. Sperm membrane functionality in the dog assessed by flow cytometry. Reprod. Domest. Anim., 47(1):39-43, 2012.

Garner, D. L.; Pinkel, D.; Johnson, L. A. \& Pace M. M. Assessment of spermatozoal function using dual fluorescent staining and flow cytometric analyses. Biol. Reprod., 34(1):127-38, 1986.

Garner, D. L. \& Johnson, L. A. Viability assessment of mammalian sperm using SYBR-14 and propidium iodide. Biol. Reprod., 53(2):276-84, 1995.

Harrison, R. A. \& Vickers, S. E. Use of fluorescent probes to assess membrane integrity in mammalian spermatozoa. J. Reprod. Fertil., 88(1):343-52, 1990.

Kim, S. H.; Yu, D. H. \& Kim Y. J. Apoptosis-like change, ROS, and DNA status in cryopreserved canine sperm recovered by glass wool filtration and Percoll gradient centrifugation techniques. Anim. Reprod. Sci., 119(1-2):106-14, 2010a.

Kim, S. H.; Yu, D. H. \& Kim Y. J. Effects of cryopreservation on phosphatidylserine translocation, intracellular hydrogen peroxide, and DNA integrity in canine sperm. Theriogenology, 73(3):282-92, $2010 \mathrm{~b}$.

Kutzler, M. A. Semen collection in the dog. Theriogenology, 64(3):747-54, 2005.

Martinez-Pastor, F.; Johannisson, A.; Gil, J.; Kaabi, M.; Anel, L.; Paz, P. \& Rodriguez-Martinez, H. Use of chromatin stability assay, mitochondrial stain JC-1, and fluorometric assessment of plasma membrane to evaluate frozen-thawed ram semen. Anim. Reprod. Sci., 84(1-2):121-33, 2004.

Nagy, S.; Jansen, J.; Topper, E. K. \& Gadella, B. M. A triple-stain flow cytometric method to assess plasma and acrosome membrane integrity of cryopreserved bovine sperm immediately after thawing in presence of egg-yolk particles. Biol. Reprod., 68(5):1828-35, 2003.

Olfert, E.; Cross, B. \& McWilliam, A. Guide to the care and use of experimental animals. Montreal, Canadian Council on Animal Care, 1998.
Peña, A.; Johannisson, A. \& Linde-Forsberg, C. Post-thaw evaluation of dog spermatozoa using a new triple fluorescent staining and flow cytometry. Theriogenology, 52(6):965-80, 1999.

Peña, F. J.; Nuñez-Martínez, I. \& Morán, J. M. Semen technologies in dog breeding: an update. Reprod. Domest. Anim., 41(Suppl. 2):21-9, 2006.

Pintado, B.; de la Fuente, J. \& Roldan, E. R. Permeability of boar and bull spermatozoa to the nucleic acid stains propidium iodide or Hoechst 33258, or to eosin: accuracy in the assessment of cell viability. J. Reprod. Fertil., 118(1):145-52, 2000.

Rijsselaere, T.; Van Soom, A; Maes, D. \& de Kruif, A. Use of the Sperm Quality Analyzer (SQA-IIC) for the assessment of dog sperm quality. Reprod. Domest. Anim.,37(3):158-63, 2002.

Rijsselaere, T.; Van Soom, A.; Maes, D.; Verberckmoes, S. \& de Kruif, A. Effect of blood admixture on in vitro survival of chilled and frozen-thawed canine spermatozoa. Theriogenology, 61(7-8):1589-602, 2005.

Risopatrón, J.; Catalán, S.; Veuthey, C.; Miska, W.; Schill, W. B. \& Sánchez, R. Effect of albumin and polyvinyl alcohol on vitality, motility and acrosome integrity in caninespermatozoa incubated in vitro. Reprod. Domest. Anim., 37(6):347-51, 2002.

Salinas, P.; Sanchez, R. \& Risopatron, J. Cryopreservation of canine spermatozoa at-80 C. Int. J. Morphol., 31(1):217-24, 2013.

Schürmann, A.; Axer, H; Scheepers, A.; Doege, H. \& Joost, H. G. The glucose transport facilitator GLUT8 is predominantly associated with the acrosomal region of mature spermatozoa. Cell Tissue Res., 307(2):237-42, 2002.

Silva, P. F. \& Gadella, B. M. Detection of damage in mammalian sperm cells. Theriogenology, 65(5):958-78, 2006.

Volpe, S.; Leoci, R.; Aiudi, G. \& Lacalandra, G. M. Relationship between motility and mitochondrial functional status in canine spermatozoa. Reprod. Domest. Anim.,44(Suppl. 2):275-8, 2009.

Yaniz, J. L.; Palacín, I.; Vicente-Fiel, S.; Gosalvez, J.; LópezFernandez, C. \& Santolaria, P. Comparison of membranepermeant fluorescent probes for sperm viability assessment in the ram. Reprod. Domest. Anim., 48(4):598-603, 2013.

\section{Dirección para Correspondencia: \\ Paulo Salinas Pérez \\ Laboratorio de Anatomía Veterinaria \\ Escuela de Medicina Veterinaria \\ Universidad Santo Tomás \\ Rodríguez 060, Temuco \\ CHILE}

Email: psalinas@santotomas.c

Recibido : 24-11-2013

Aceptado: 23-01-2014 